\begin{tabular}{|c|c|c|c|c|}
\hline 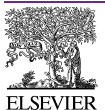 & Opinion & TRENDS in Ecology and Evolution & Vol.19 No.2 February 2004 & $\begin{array}{l}\text { Full text provided by www.sciencedirect.com } \\
\text { science DiRECT. }\end{array}$ \\
\hline
\end{tabular}

\title{
A major evolutionary transition to more than two sexes?
}

\author{
Joel D. Parker \\ Department of Ecology and Evolution, University of Lausanne, BB, CH-1015, Lausanne, Switzerland
}

Two recently discovered cases of genetic caste determination in social insects might provide the first example of a major evolutionary transition from two to more than two sexes. I argue here that the system can be interpreted as comprising primarily individuals requiring gametes from three parental types and having four sexes from the perspective of demographic extinction. Additionally, I show how this mating system can be seen as a major evolutionary transition. For these populations, it is apparent that the mechanism for a three- or four-sex system does not lie within the myriad of possible arrangements of chromosomes within individuals, but at the next level of evolutionary complexity, with the arrangement of chromosomes among individuals within a social system.

Fisher's observation that 'the sexes are, in fact always two' might have been premature [1]. Two recently discovered systems within the ant genus Pogonomyrmex (one in $P$. rugosus, the other in $P$. barbatus) each comprise two distinct types of female and two distinct types of male [2-6]. The most recent study of these populations establishes that they are not classic hybrid zones, but autonomous and perpetually hybridizing systems [6] (Box 1). Although this continuous and caste-specific type of HYBRIDIZATION (see Glossary) was originally termed 'social HYBRIDOGENESIS' [3,4], I prefer the term 'symmetrical social hybridogenesis' ( $\mathrm{SSH}$ ) to distinguish it from other social insect cases where hybrids are directed to workers in only one direction, as for some fire ant hybrid zones [7]. Two such complete systems have been discovered [6] (contrary to one suggestion of three [8]), one in a population of $P$. rugosus [4] and another in P. barbatus [2,3]. Unfortunately, there are not yet ideal taxonomic names for these two SSH populations so each case is still classified as the species that it most closely resembles morphologically (either P. rugosus or P. barbatus).

To date, the literature has emphasized either the genetic CASTE DETERMINATION [2-5] or the population genetics of the systems $[4,6]$, but has not addressed the broad implications of SSH reproduction. Not only can most of the individuals in these populations be seen as arising from the union of at least three gamete types, but all four gamete types are also necessary for the population to persist. This system is based upon a novel organization of genetic information within the insect societies and populations that qualifies as a major evolutionary transition (Box 2).

\section{Defining number of sexes of a system}

Before trying to count the number of sexes in a $\mathrm{SSH}$ population, one needs to understand exactly what defines sex and the number of sexes in a mating system. The diversity of sexual organisms and their mating systems makes a single straightforward definition difficult. The simplest definition is to count the number of gametic types, which would result in thousands for some organisms, such as the mushroom Corpinus cinereus $[9,10]$. However, simply counting the number of parts and ignoring the nature of the connections within the system misses the

\begin{abstract}
Glossary
Caste determination: the process that determines which functional class an individual will develop into. In the symmetrical social hybridogenesis (SSH) populations, I refer more specifically to reproductive caste determination or the process that determines whether a fertilized egg becomes a fertile queen or a sterile worker. Usually, this is determined by environmental factors, as in honeybees, where queens only develop when fed royal jelly.

Crossing over: the process whereby two homologous strands of DNA exchange sections before the formation of gametes during meiosis. Dominance interactions: the interaction of alternate alleles of a gene in a diploid or polyploid organism. One allele might dominate the phenotype in the heterozygous state whereas the other is recessive, only expressing a recessive phenotype in the absence of the dominant allele. Alternately, combinations of some alleles might have greater impact than either allele separately.

Epistatic interactions: interactions among genes from different loci effecting phenotype. This occurs when the phenotype of a particular gene depends upon the genetic background of the individual.

Heterosis: when a hybrid is more vigorous than either parental line (hybrid vigor) owing to an increase in heterozygosity. In the SSH case, the mixed gene pool workers might be physiologically more competitive owing to their mixed parentage in comparison to either ancestral pure strain workers.

Hybridization: interbreeding of two species or genetically different groups. These crosses are usually infertile; however, viable offspring are sometimes produced, resulting in significant gene flow among parental groups.

Hybridogenesis: a reproductive system of females arising from a hybridization event where the parental line male genome is systematically excluded from the germ line of the progeny. These species are functionally clonal. In fishes, when the sperm of the parental line is only used for activating an egg, the condition is termed gynogenesis.

Recombination: any process that generates new combinations of genes within individuals. This is usually thought of in terms of independent assortment of chromosomes or crossing over within germ line cells to create new combinations of genes.

Superorganism: a group of individuals that are selected to some extent at the level of the group and share a functional organization with traditional organisms. The concept was originally dismissed because it was based too much on group selection, but has been making a comeback with our increasing understanding of multilevel selection $[13,17]$. The most well known examples are colonies of social insects, such as the honey bee, comprising sterile workers carrying out specialized tasks and one reproductive female.
\end{abstract}




\section{Box 1. Mating patterns in symmetrical social hybridogenesis social insect populations}

In normal haplodiploid eusocial insect systems, haploid males arise parthenogenically from unfertilized eggs. Fertilized diploid eggs develop into one of the two female castes (worker or reproductive female) that are determined by some environmental factors (Figure la). In symmetrical social hybridogenesis (SSH) systems, females with parents from the same gene pool become queens (blue or yellow females in Figure I), whereas fertilized eggs of mixed gene pool parentage become workers (green sterile workers in Figure I). Males arise from unfertilized eggs and are of the same gene pool as their mother.

The genetic determination of caste in SSH systems means that, when a queen mates exclusively with males from the same gene pool, she cannot produce workers, and colony founding is therefore impossible. If a queen mates only with males from the opposite gene pool, workers are produced and colony founding can occur. These colonies can become male-only producing colonies because haploid males come from unfertilized eggs. Only colonies with queens who have mated with both types of male can produce both workers and two types of reproductive sexuals. These are hermaphrodites from the superorganism perspective, producing eggs from the queen and sperm in the case of the haploid male offspring. Most of the colonies observed in the field are of this multiply mated type, but a few male-only colonies have been observed [4]. The SSH breeding system separates the gene pools because the only hybrid individuals are sterile workers. Some virgin queens of opposite gene pool crosses are produced (not shown in Figure I), but they are not viable, as evident from the lack of observable gene flow across the gene pools $[4,6]$.

The unique production of sterile individuals from opposite type crosses requires a novel terminology to classify the gametes of these systems. I use the term 'gametic type' to refer to the four classes of gametes (sperm from yellow pool males, sperm from blue sperm males, eggs from yellow pool queens and eggs from blue pool queens in Figure I). This closely follows Hoekstra [16], using mating types to designate two sexes without morphological differentiation. In other systems, mating types are defined in terms of pre-fertilization incompatibilities unlike the situation here [16].

(a)

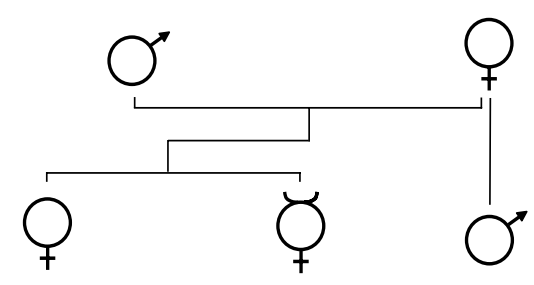

(b)

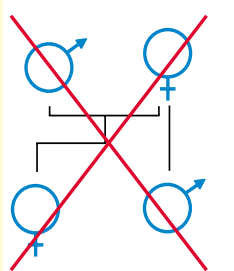

No workers, colonies die

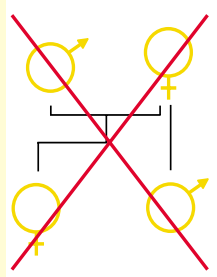

No workers, colonies die
Blue queen colonies

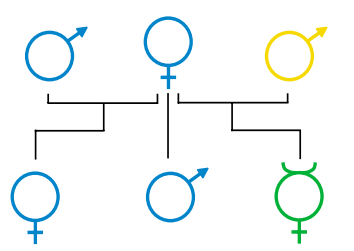

Female- and male-producing colonies

Yellow queen colonies

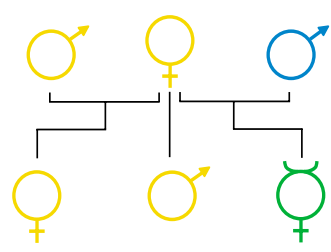

Female- and male-producing colonies

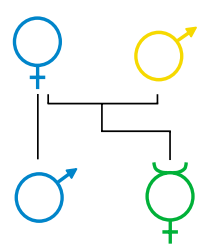

Male-producing colonies

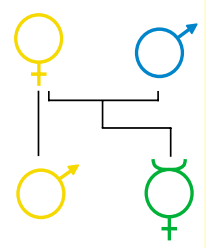

Male-producing colonies
$\overline{T R E N D S}$ in Ecology \& Evolution

Figure I. Mating structure of typical and symmetrical social hybridogenesis (SSH) haplodiploid eusocial systems. (a) depicts the typical situation, in which fertilized eggs become female [queens (female symbol) or workers (Hermes, intersex symbol)] depending on environmental cues. By contrast, (b) shows that, when caste is genetically determined, four viable colony types can exist owing to the requirement for workers. Blue and yellow indicate the two isolated gene pools and green indicates individuals that are a mix of the two. The two rows show the mating structures of colonies headed by blue gene pool queens on top and yellow gene pool queens on bottom in order, depending on the combinations of males that the queens mate with by chance. The red $X$ indicates that colony founding will fail owing to the lack of workers if the queen only mates with same gene pool males. In the middle are queens mated with males from both gene pools and, on the right are queens that are mated only with males from the opposite gene pool. The gene pools shown in Figure I correspond to the lineages ( $\mathrm{H} 1$ and $\mathrm{H} 2$, or $\mathrm{J} 1$ and $\mathrm{J} 2$ ) in Helms Cahan [6]. Thus, for the Pogonomrymex rugosus case [4], the lineages $\mathrm{H} 1$ and $\mathrm{H} 2$ can be thought of as corresponding to the blue and yellow pools in Figure I, or in the $P$. barbatus case $[2,3]$, the lineages $\mathrm{J} 1$ and $\mathrm{J} 2$ can be thought of corresponding to the blue and yellow pools. whole point of why sexual systems appear to be a binary process at some fundamental level.

Perhaps the most popular functional definition for why sex is binary is the process of CROSSING OVER. Clearly, crossing over is one of the factors contributing to the success of sex, but are they actually one and the same? Even those advocating a primary role for RECOMBINATION are careful not to define sex as crossing over among chromosomes but as involving 'the coming together of genetic material from two parents to form progeny that combine genes from both of them' [11]. Even here, the binary quality is present in the explicit requirement of two parents. A more open, general and equally intuitive functional definition is to count the number of gamete types that must unite to form any of the fertile individuals in a population. Under this definition, a system with more than two sexes would require that some individuals arise from more than two distinct classes of gametes. Counting the number of gamete types per individual not only includes crossing over as a special case, but also opens 


\section{Box 2. The big picture}

- A mating system that evolved twice through hybridization in the seed harvester ant Pogonomyrmex, where queens must mate with two distinct types of male to produce a colony with both sterile workers and fertile females, can be considered a system with more than two sexes, proving that Fisher was premature in concluding that 'the sexes, are in fact always two'.

- Any colony that makes a fertile queen must have at least three parents, or be descendent from three distinct and separate types of gamete.

- Four separate types of gamete must be maintained in the population or the population will become extinct.

- The system constitutes a major evolutionary transition under the criteria of Szathmáry and Maynard Smith.

- Thus, I argue that a more than two sex system has evolved through hybridization of two previously autonomous social insect populations. It appears that evolution to more than two sexes depends upon a higher order of organization of genetic information within a social population.

up the possibility that crossing over is a property of twosex systems and not necessarily fundamental to three- or more sex systems.

Another approach is to consider the stability of a sexual system. The definition of sexes that is implicit in the concept of demographic extinction captures this second idea. Demographic extinction occurs when the population goes extinct owing to a lack of one sex. The number of demographic sexes in a population can be thought of as the greatest number of exclusive groups of sexes where loss of any single group will cause extinction of the entire system. If we apply this definition at the level of gamete types, then all previously known sexual populations, to my knowledge, reduce to two-sex systems. For example, sequentially and permanently removing mating types from a system with many mating types would not result in the extinction of the remaining members of the population until one of the last two reproducing self-incompatible types is removed. In the case without self-incompatibility, the two groups would be the very last two gametes remaining.

A system with more than two sexes would require either more than two groups of gametes to produce a fertile individual by the functional definition, and/or more than two groups of gametes for the population to persist by the stability definition. These definitions are independent and a population might satisfy only one of these definitions or might even be classified differently as to number of sexes, depending on which definition is used.

\section{Three parents make one individual}

Social insect colonies have been considered as examples of SUPERORGANISMS because they share organization that is similar to that of solitary organisms [12]. Most notable is the division of reproductive labor, where the only fitness of sterile workers is through their indirect relationship to the reproductives produced by the queen. Hence, worker fitness is primarily determined by the colony fitness. Indeed, highly developed eusocial insects, such as those discussed here, have been recently viewed as superorganisms in spite of well known conflicts within colonies [13]. From this perspective, colonies in SSH populations are a three-sex system of four reproductive types; two types that produce only males, and two types that produce both male and females (Box 1, Figure I).

If a queen mates by chance exclusively with males from the opposite gene pool, she can only make workers and parthenogenically produced haploid males. However, the colonies that produce both males and females, which can be considered hermaphrodites, require three gametic types (sperm from both the yellow and blue gene pools and egg from the queen; Box 1, Figure I). The male gamete from the opposite gene pool is directed to the soma, or body of the colony (i.e. the workers), whereas the male gamete from the same gene pool as the queen contributes to the female reproductives. The third gamete type is then the egg of the queen. Only colonies that are descended from this union of the three gametic types can produce viable female reproductive offspring. These colonies producing two sexes are hermaphroditic individuals and can only result from the contribution of three different types of gamete and have a minimum of three parents when selfing is excluded, thus satisfying the functional definition of a three-sex system.

\section{Four sexes required for persistence}

The obligatory interdependence of gametic types across the two gene pools also has important ramifications for the persistence of the population as a whole. According to the stability definition above, to classify a system with more than two sexes, the gametes must be divisible into more than two independent sets, such that the loss of any one of the sets results in the extinction of the entire system. SSH populations meet this criterion. Losing any one of the four gametic types (yellow sperm or egg, blue sperm or egg in Figure I, Box 1) will result in the collapse of the entire system. This is due to the loss of a queen type indirectly, or the loss of workers in one colony type owing to the lack of sperm from the opposite gene pool. Thus, the system is completely dependent on exactly four gametic types and hence can be classified as four sex by the demographic extinction definition.

I know of no other systems of mating incompatibilities or other cases of hybridogenesis where the gametes can be put into more than two groups and loss of any one of these groups will lead to the demographic extinction of the entire system. If other such systems exist, they too might be systems with more than two sexes.

\section{A major evolutionary transition}

The concept of major evolutionary transitions was invented, in part, to characterize more accurately the evolution of sex [14]. Thus, it might be expected that the evolution of a system with more than two sexes would constitute a major evolutionary transition. In fact, the transition to SSH can be considered a major evolutionary transition as defined by the information theory of Szathmáry and Maynard Smith [14]. They list three features that major evolutionary transitions share: (i) replicating units that existed before the transition can only replicate as parts of larger units after the transition; (ii) an increase in the division of labor; and (iii) changes in information storage and transmission. $\mathrm{SSH}$ satisfies all three criteria. 
First, two previously autonomous populations or gene pools have united into one interacting, mutually interdependent pair, as recently confirmed with the determination of the hybrid origins of the populations [6]. The system is irreversible, because pure-type queens require other type males to make workers, and the lack of observable gene flow among the gene pools proves that hybrid queens are not viable [4,6]. Irreversibility is also supported by the observation of no gene flow with the putative ancestral hybridizing species [6]. The second requirement for an increase in division of labor is satisfied at the genotypic level. This involves the division of EPISTATIC and DOMINANCE INTERACTIONS because some combinations of alleles are limited to the yellow gene pool and others to the blue gene pool in sexuals (Box 1, Figure I). This includes epistasis among genes, interactions among cytoplasmic factors and nuclear genes, and dominance interactions among alleles. Inter-gene pool interactions of the three kinds can occur only in the sterile workers. Thus, not only are epistasis and dominance interactions separated and maintained by the lack of gene flow among the gene pool units, but they are also overlaid onto the existing division of reproductive labor. Perhaps the most important example is the partitioning of possible hybrid sterility (and potential HETEROSIS) to workers who are already infertile. The implications and exact nature of this division is currently unknown and should be a fruitful area for future work as it is the most probable source of the success of the species. Third, information is stored and transferred in a different and more complex structure, in two separate pools of genes, satisfying the third criteria for a major transition. Finally, this transition to SSH could have evolved only from eusociality, which was previously regarded as one of the last major transitions.

\section{Conclusion}

SSH therefore constitutes a major evolutionary transition and comprises more than two sexes from the perspective of the number of gametic types required to make individuals in the population (functional definition) and the number required to prevent demographic extinction (stability definition). The observation that crossing over is still limited to two genomes even when three types of gametes must combine to make most of the fertile individuals suggests that crossing over is a property that characterizes the transition to two sexes, and is not necessarily important for the transition to systems with more than two sexes. Indeed, SSH systems might be a good testing ground to assess the relative importance of recombination [11] and cytoplasmic incompatibility theories [15] for the evolution of sex and the sexes.

From the stability or demographic point of view, this major transition has doubled the number of sexes by combining two extant independent species into one coherent more complex whole that now depends on retaining all of the ancestral sexes. Thus, these three or four sex populations solve the question that so puzzled Fisher and many others. The answer to how a system with more than two sexes works is to consider the phenomenon at a level above the individual. SSH results in a higher level of recombinational complexity based on rules for shuffling chromosomes within a social system rather than within individual organisms or cells. This is not recombination in the sense of crossing over of DNA molecules, but a new set of rules for how gene pools are partitioned within a population, and how chromosomes are arranged among classes of individuals in social groups.

\section{Acknowledgements}

I thank Michel Chapuisat, Philippe Christe, Tom Dowling, Jennifer Fewell, Rob Hammond, Sara Helms Cahan, Kimberly Hughes, Stephanie Jemielity, Laurent Keller, Karen Parker, Steve Rissing, Eörs Szarmarthy, four anonymous referees and the Social Insect Research Group at ASU for comments and key criticisms. Article preparation was supported by Laurent Keller with grants from The AETAS Foundation, The Fondation A. R. and J. Leenards (Lausanne) and The Swiss National Science Foundation.

\section{References}

1 Fisher, R.A. (1930) The Genetical Theory of Natural Selection, Clarendon Press

2 Volny, V.P. and Gordon, D.M. (2002) Genetic basis for queen-worker dimorphism in a social insect. Proc. Natl. Acad. Sci. U. S. A. 99, 6108-6111

3 Julian, G.E. et al. (2002) Genetic determination of the queen caste in an ant hybrid zone. Proc. Natl. Acad. Sci. U. S. A. 99, 8157-8160

4 Helms Cahan, S. et al. (2002) Extreme genetic differences between queens and workers in hybridizing Pogonomyrmex harvester ants. Proc. R. Soc. Lond. Ser. B 269, 1871-1877

5 Ashe, A. and Oldroyd, B. (2002) Genetic determination of caste in a social insect. Trends Ecol. Evol. 17, 448-449

6 Helms Cahan, S. and Keller, L. (2003) Complex hybrid origin of genetic caste determination in harvester ants. Nature 424, 306-309

7 Helms Cahan, S. and Vinson, B. (2003) Reproductive division of labor between hybrid and non-hybrid offspring in a fire ant hybrid zone. Evolution 57, 1562-1570

8 Ashe, A. and Oldroyd, B. (2003) Do queens select sperm? Trends Ecol. Evol. 18, 108

9 Raper, J.R. (1966) Genetics of Sexuality in Higher Fungi, The Ronald Press Company

10 Cassleton, L.A. (2002) Mate recognition in fungi. Heredity 88, 142-147

11 Barton, N.H. and Charlesworth, B. (1998) Why sex and recombination? Science 281, 1986-1990

12 Wilson, D.S. and Sober, E.J. (1989) Reviving the superorganism J. Theor. Biol. 136, 337-357

13 Queller, D.C. and Strassmann, J.E. (2002) The many selves of social insects. Science 296, 311-313

14 Szathmáry, E. and Maynard Smith, J. (1995) The major evolutionary transitions. Nature 374, 227-232

15 Hurst, L.D. and Hamilton, W.D. (1992) Cytoplasmic fusion and the nature of the sexes. Proc. R. Soc. Lond. Ser. B 247, 189-194

16 Hoekstra, R.F. (1987) The evolution of sexes. In The Evolution of Sex and its Consequences (Stearns, S.C., ed.), pp. 59-91, Birkhauser Verlag

17 Seely, T.D. (1997) Honey bee colonies are group-level adaptive units. Am. Nat. 150, s22-s41 